\title{
Frequência à puericultura por crianças de 0-2 anos da cidade de Rio Branco-Acre e suas consequências
}

\author{
Childcare attendance by children aged 0-2 years in the city of Rio Branco-Acre and its \\ consequences
}

\author{
Asistencia al cuidado de niños de 0-2 años en la ciudad de Rio Branco-Acre y sus \\ consecuencias
}

\begin{abstract}
Thatyana Frias Freitas ${ }^{1 *}$, Carolina Pinho de Assis Pereira Roque ${ }^{1}$, Thaís Jardim Teodoro Branco ${ }^{1}$, Bárbara Bueno Manzati ${ }^{1}$, Ariovaldo Manzati Junior ${ }^{1}$.
\end{abstract}

\begin{abstract}
RESUMO
Objetivo: Objetivou-se avaliar a frequência à puericultura por crianças de Rio Branco, bem como os seus componentes e consequências da não adesão. Métodos: Trata-se de estudo descritivo transversal, observacional, quantitativo, composto por diversas variáveis (assistência à saúde, evidências epidemiológicas, morbidades, internações e mortalidade), sendo dados coletados no Departamento de Informática do Sistema Único de Saúde do Brasil. Resultados: Nota-se que o valor médio de consultas de puericultura corresponde apenas a $6,5 \%$ das que deveriam ocorrer anualmente. Dentre os menores de 1 ano e entre 12-23 meses observa-se poucas com calendário vacinal atualizado, baixo acompanhamento do peso, alta mortalidade por desnutrição e pneumonia, baixa prevalência do aleitamento materno exclusivo e muitas internações por infecções respiratórias. Conclusão: Implantação da puericultura trouxe resultados positivos ao avaliar a saúde infantil no Brasil. Porém muitas Unidades Básicas de Saúde não usam tal ferramenta adequadamente, o que prejudica a melhora dos indicadores de saúde infantil e mortalidade, tornando assim um país heterogéneo que é evidenciado em diversos estudos já realizados. Concernente a isto, evidenciou-se que no Acre também há uma diversidade entre seus Municípios e que, Rio Branco como capital, ainda deixa a desejar no quesito de cobertura da puericultura, sendo muito aquém do ideal.
\end{abstract}

Palavras-chave: Cuidado da criança, Crescimento e desenvolvimento, Mortalidade infantil, Atenção primária à saúde.

\begin{abstract}
Objective: The objective was to evaluate the frequency of childcare by children from Rio Branco, as well as its components and consequences of non-adherence. Methods: This is a descriptive, cross-sectional, observational, quantitative study, composed of several variables (health care, epidemiological evidence, morbidities, hospitalizations and mortality), with data collected at the Informatics Department of the Brazilian Unified Health System. Results: It is noted that the average value of childcare consultations corresponds only to $6.5 \%$ of those that should occur annually. Among those younger than 1 year and between $12-23$ months, there are few with an updated vaccination schedule, low weight monitoring, high mortality from malnutrition and pneumonia, low prevalence of exclusive breastfeeding and many hospitalizations for respiratory infections. Conclusion: Implementation of childcare brought positive results when assessing child health in Brazil. However, many Basic Health Units do not use this tool properly, which hinders the improvement of child health and mortality indicators, thus making it a heterogeneous country that is evidenced in several studies already carried out. Concerning this, it was evidenced that in Acre there is also a diversity among its Municipalities and that, Rio Branco as capital, still leaves something to be desired in terms of childcare coverage, being far from ideal.
\end{abstract}

Keywords: Child care, Growth and development, Infant mortality, Primary health care.

${ }^{1}$ Centro Universitário Uninorte, Rio Branco - AC. “E-mail: thatyana.ff96@gmail.com

SUBMETIDO EM: 9/2020

ACEITO EM: 10/2020

PUBLICADO EM: 11/2020

REAS/EJCH | Vol.12(11) | e5177 | DOI: https://doi.org/10.25248/reas.e5177.2020 Página 1 de 11 


\section{RESUMEN}

Objetivo: El objetivo fue evaluar la frecuencia del cuidado infantil por parte de los niños de Rio Branco, así como sus componentes y consecuencias de la no adherencia. Métodos: Se trata de un estudio descriptivo, transversal, observacional, cuantitativo, compuesto por varias variables (atención de salud, evidencia epidemiológica, morbilidades, hospitalizaciones y mortalidad), con datos recolectados en el Departamento de Informática del Sistema Único de Salud Brasileño. Resultados: Se observa que el valor promedio de las consultas de puericultura corresponde solo al $6.5 \%$ de las que deben ocurrir anualmente. Entre los menores de 1 año y entre 12 y 23 meses, son pocos los que tienen un calendario de vacunación actualizado, control de bajo peso, alta mortalidad por desnutrición y neumonía, baja prevalencia de lactancia materna exclusiva y muchas hospitalizaciones por infecciones respiratorias. Conclusión: La implementación del cuidado infantil arrojó resultados positivos al evaluar la salud infantil en Brasil. Sin embargo, muchas Unidades Básicas de Salud no utilizan adecuadamente esta herramienta, lo que dificulta la mejora de los indicadores de salud y mortalidad infantil, lo que lo convierte en un país heterogéneo como se evidencia en varios estudios ya realizados. Al respecto, se evidenció que en Acre también hay diversidad entre sus Municipios y que, Rio Branco como capital, aún deja algo que desear en cuanto a cobertura de cuidado infantil, distante de ser ideal.

Palabras clave: Cuidado infantil, Crecimiento y desarrollo, Mortalidad infantil, Atención primaria de salud.

\section{INTRODUÇÃO}

A criança faz parte do grupo prioritário de atenção à saúde, o que pressupõe garantir a implementação de uma assistência que favoreça o processo do cuidado de tal faixa etária. A Política Nacional de Atenção Básica (PNAB) define a Atenção Primária à Saúde (APS) como o nível de atenção à saúde que representa o primeiro contato com as crianças e familiares oferecendo serviços e ações que visam a atenção integral à criança com ações voltadas para promoção e reabilitação da saúde, prevenção e cura de agravos, garantindo assim a longitudinalidade do cuidado (HANZEN IP, et al., 2019).

Para isso, desenvolveu-se o programa de puericultura que tem como objetivo a realização da avaliação integral da criança, abordando os aspectos de crescimento, desenvolvimento neuropsicomotor, vacinação, intercorrências, estado nutricional, orientações à mãe/família/cuidador em todos os atendimentos, de modo que a criança alcance a vida adulta sem influências desfavoráveis decorrentes da infância (BRASIL, 2005). Ressaltando-se que há necessidade de avaliar a criança não só no contexto ambulatorial, como também diante do contexto socioeconômico, cultural e familiar que está inserida (FALBO BC, et al., 2012).

Um dos objetivos mundiais vem a ser a diminuição da mortalidade infantil. Com isso, observou-se que grande parte dos problemas de saúde da criança podem ser enfrentados através de ações, como a puericultura, que gerem mudanças significativas nos indicadores de saúde e mortalidade infantil (VIEIRA GO, 2017).

Após implantação da puericultura, observou-se queda de $53 \%$ da mortalidade infantil mundial, tendo reduzido de 91 para 43 mortes por 1.000 nascidos vivos. Porém, nem toda a população partilha de tal avanço, permanecendo a vulnerabilidade maior dos grupos mais pobres em relação aos mais ricos. Grande parte desses óbitos podem ser evitados com medidas simples, como vacinação, amamentação, higiene, acesso a medicamentos, dentre outros fatores que estão inclusos na puericultura (BRASIL, 2005; TEJADA CA, et al., 2019).

A média brasileira de mortalidade infantil, em 2018, encontrava-se entre 12,17 óbitos por 100 nascidos vivos, porém muitas cidades e estados estão acima disso. Concomitantemente, no Maranhão, taxa de mortalidade infantil permanece elevada, 14,07\% (CUNHA CL, et al., 2013; BRASIL, 2020).

Corroborando para isso temos o percentual de crianças maranhenses com esquema vacinal básico, aleitamento materno exclusivo (AME) e cobertura de consultas inferior à média nacional (TAVARES MN, et al., 2019).

Ressalta-se que existem lacunas na realização das consultas de puericultura, dentre elas a falta de conhecimento tanto de profissionais como de pais/responsáveis sobre a importância do acompanhamento do crescimento e desenvolvimento infantil; falta de acompanhamento da situação vacinal; além da dificuldade de 
implementação de um cuidado sistematizado. Desta forma a consulta médica passa a ter foco curativista, enquanto que deveria ter foco preventivo. A cerca disso observa-se a importância do acompanhamento por meio do programa de puericultura como importante ferramenta ao combate de agravos evitáveis que acometem tal faixa etária da população, como diarreia, desnutrição, pneumonia, bronquite e bronquiolite agudas (TAVARES MN, et al., 2019).

Diante do exposto, objetivou-se, através deste estudo, avaliar a frequência à puericultura por crianças residentes em Rio Branco, bem como os seus componentes e consequências da não adesão.

\section{MÉTODOS}

Trata-se de estudo descritivo transversal, observacional, quantitativo, em Rio Branco-Acre. A população do estudo foi composta por diversas variáveis notificadas relacionadas ao tema abrangente de puericultura, ocorridos em Rio Branco, de 2001-2019.

Os dados desta pesquisa foram coletados no Departamento de Informática do Sistema Único de Saúde do Brasil (DATASUS), em março de 2020, submetidos ao cálculo de frequência absoluta, relativa, razões de prevalência e tabulados pelo programa Microsoft Excel 2016.

Dentre as variáveis analisadas encontram-se fatores relacionados à assistência à saúde referindo-se à APS, como óbito por diarreia, infecção respiratória aguda (IRA) e outras causas em < 28 meses, 1 ano e 2 anos; aleitamento materno em $<4$ meses; total da população de $<1$ ano e entre 12-23 meses, vacinas em dia, pesadas e desnutridas de tais faixas etárias) e produção ambulatorial com puericulturas apresentadas.

Outro fator levantado enquadrou-se entre as evidências epidemiológicas e morbidades, quanto à morbidade hospitalar do Sistema Único de Saúde (SUS), internações e taxa de mortalidade referente a desnutrição, diarreia, além de internações por IRA.

Elencaram-se os dados disponíveis quanto as estatísticas vitais abordando óbitos por causas evitáveis entre 0-4 anos (como diarreia, desnutrição, pneumonia, bronquite e bronquiolite agudas) e nascidos vivos.

\section{RESULTADOS E DISCUSSÃO}

No Brasil, a puericultura foi implementada a PNAB e concretiza-se através do acompanhamento periódico e sistemático das crianças para avaliação de seu crescimento e desenvolvimento, vacinação, orientações, aleitamento materno (AM), higiene e identificação precoce de agravos, com intuito de realizar intervenções. A APS passou a representar o primeiro contato com as crianças, oferecendo serviços e ações de atenção integral (BRASIL, 2005; HANZEN IP, et al., 2019; CAMPOS RM, et al., 2011).

A infância torna-se o período da vida de maior preocupação quanto ao desenvolvimento. Para a Organização Mundial de Saúde (OMS), o desenvolvimento infantil trata-se do processo que ocorre desde a concepção do feto, envolvendo crescimento físico, maturação neurológica, comportamental, cognitiva, social e afetiva, sendo mais susceptível às doenças ou agravos (SANTOS ME, et al., 2010).

Durante as consultas de puericultura deve-se sempre enfatizar e esclarecer que a promoção da saúde não é apenas responsabilidade do profissional, mas também de todos os envolvidos no cuidado, comprometendose em prestar assistência ao infante estando sadio ou doente (VASCONCELOS VM, et al., 2012).

De acordo com a Sociedade Brasileira de Pediatria, a puericultura volta-se para acompanhamento de indivíduos entre 0-19 anos observando-se algumas particularidades (Tabela 1). Levando-se em consideração a faixa etária da puericultura e a quantidade de consultas preconizadas, evidencia-se que cada indivíduo, durante sua vida, deveria passar por no mínimo 31 consultas destinadas para puericultura. Analisando mais especificamente para a faixa etária do estudo, observou-se que a quantidade mínima de consultas na faixa etária de 0-2 anos corresponde a 12 (SOCIEDADE BRASILEIRA DE PEDIATRIA, 2017). 
Tabela 1 - Consultas de puericultura conforme idade.

\begin{tabular}{ccc}
\hline Classificação etária & Idade & Acompanhamento \\
\hline RN & 0 à 28 dias. & $\begin{array}{c}\text { Visita domiciliar na primeira semana e deve-se marcar a } \\
\text { primeira consulta no programa. }\end{array}$ \\
\hline Lactente & 28 dias à 24 meses. & $\begin{array}{c}\text { Mensal até o sexto mês, trimestral a partir de então até } \\
\text { o décimo oitavo mês e semestral a partir de 18 meses. }\end{array}$ \\
\hline Pré-escolar & 2 à 4 anos. & Semestral. \\
\hline Escolar & 5 à 10 anos. & Anual. \\
\hline Adolescente & 11 à 19 anos. & Anual.
\end{tabular}

Fonte: FREITAS TF, et al., 2020; dados extraídos da SOCIEDADE BRASILEIRA DE PEDIATRIA, 2017; CIAMPO LA, et al., 2006.

Levantou-se dados quanto a quantidade de puericulturas apresentadas entre 2008-2018 e observou-se que a média esteve em torno de 13.580 consultas/ano. Com isso, foi realizada comparação do valor médio de consultas com a média de nascidos vivos de tais anos (Tabela 2). Ao realizar o produto da média de nascidos vivos (6.740) pela quantidade de consultas mínimas (31), verificou-se que o valor médio de consultas deveria se aproximar de 208.940 em relação a todos os nascidos vivos/ano. Nota-se que o valor médio corresponde apenas a $6,50 \%$ do estimado de consultas/ano de puericulturas.

Tabela 2 - Puericulturas apresentadas, nascidos vivos e a média, no município de Rio Branco, 2008-2018.

\begin{tabular}{ccc}
\hline Ano & Puericulturas apresentadas & Nascidos vivos \\
\hline 2008 & 7969 & 7068 \\
2009 & 9388 & 6538 \\
2010 & 11946 & 6437 \\
2011 & 19513 & 6902 \\
2012 & 16938 & 6531 \\
2013 & 21364 & 7116 \\
2014 & 19347 & 7011 \\
2015 & 12215 & 6965 \\
2016 & 13218 & 6614 \\
2017 & 14360 & 6460 \\
2018 & 3126 & 6495 \\
Média & 13580 & 6740 \\
\hline
\end{tabular}

Fonte: FREITAS TF, et al., 2020; dados extraídos do DATASUS.

A frequência ao programa está aquém do esperado, corroborando com estudos realizados em outras cidades. Podemos citar o Maranhão, onde evidenciou-se que a porcentagem de crianças que tiveram atendimento no primeiro mês de vida e ao final do primeiro ano foram $35,6 \%$ e $38,4 \%$ respectivamente (CUNHA CL, et al., 2013). Condizentes têm São Leopoldo, onde evidenciou-se que 53,2\% das crianças não foram acompanhadas regularmente (VITOLO MR, et al., 2010).

Afirma-se que as resistências dos familiares em participarem do programa entrelaçam-se com crenças, cultura dos idosos, desvalorização do programa, baixa escolaridade e renda, muitos filhos, curto espaço entre partos, falta de agentes comunitários e materiais (BRASIL, 2005; CUNHA CL, et al., 2013).

A imunização está entre as melhores estratégias de prevenção de morbimortalidade na infância, e seu custo-benefício supera as ações terapêuticas e de reabilitação. No entanto, em regiões mais carentes, relacionam-se várias situações, como entraves culturais, educacionais e problemas estruturais dos serviços de saúde, que prejudicam a adesão, além de que as crianças não participantes das vacinações podem ser as que não frequentam a puericultura (FERNANDES AC, et al., 2015). 
Entre 2001-2015, dentre < 1 ano e 12-23 meses, a média de vacinação em dia esteve em $89 \%$ e $88 \%$ respectivamente (Tabela 3). Ao compararmos com a cobertura vacinal considerada satisfatória pelo Ministério da Saúde (MS), 95\%, notamos que a média das vacinações dos rio-branquenses encontra-se abaixo (FERNANDES AC, et al., 2015).

Tabela 3 - População, calendário vacinal atualizado e cobertura vacinal com média, de $<1$ ano e 12 23 meses, de Rio Branco, 2001-2015.

\begin{tabular}{ccccccc}
\hline \multirow{2}{*}{ Ano } & \multicolumn{3}{c}{$<$ 1 ano } & & \multicolumn{3}{c}{$\mathbf{1 2 - 2 3 \text { meses }}$} \\
\cline { 2 - 7 } & População & $\begin{array}{c}\text { Calendário } \\
\text { vacinal } \\
\text { atualizado }\end{array}$ & $\begin{array}{c}\text { Cobertura } \\
\text { vacinal }\end{array}$ & População & $\begin{array}{c}\text { Calendário } \\
\text { vacinal } \\
\text { atualizado }\end{array}$ & $\begin{array}{c}\text { Cobertura } \\
\text { vacinal }\end{array}$ \\
\hline 2001 & 37.966 & 27.336 & $72 \%$ & 43.777 & 32.986 & $75 \%$ \\
2002 & 28.020 & 21.128 & $75 \%$ & 32.768 & 25.699 & $78 \%$ \\
2003 & 26.874 & 21.752 & $81 \%$ & 28.713 & 23.694 & $83 \%$ \\
2004 & 26.349 & 22.373 & $85 \%$ & 28.903 & 24.392 & $84 \%$ \\
2005 & 29.501 & 26.167 & $89 \%$ & 31.591 & 27.352 & $87 \%$ \\
2006 & 30.457 & 27.837 & $91 \%$ & 31.479 & 28.032 & $89 \%$ \\
2007 & 27.820 & 25.404 & $91 \%$ & 28.561 & 25.516 & $89 \%$ \\
2008 & 27.539 & 25.652 & $93 \%$ & 29.890 & 27.433 & $92 \%$ \\
2009 & 26.140 & 24.558 & $94 \%$ & 26.310 & 24.486 & $93 \%$ \\
2010 & 20.143 & 18.930 & $94 \%$ & 21.750 & 20.327 & $93 \%$ \\
2011 & 19.004 & 18.042 & $95 \%$ & 18.769 & 17.577 & $94 \%$ \\
2012 & 19.344 & 18.117 & $94 \%$ & 19.499 & 18.109 & $93 \%$ \\
2013 & 22.215 & 20.297 & $91 \%$ & 23.237 & 20.949 & $90 \%$ \\
2014 & 20.365 & 18.811 & $92 \%$ & 21.070 & 18.871 & $90 \%$ \\
2015 & 14.102 & 12.932 & $92 \%$ & 15.256 & 13.700 & $90 \%$ \\
Média & 25.056 & 21.956 & $89 \%$ & 26.772 & 23.275 & $88 \%$ \\
\hline
\end{tabular}

Fonte: FREITAS TF, et al., 2020; dados extraídos do DATASUS.

Ao analisar cada ano individualmente, atingiu-se a cobertura preconizada apenas em $2011 \mathrm{em}<1$ ano e nenhum ano de 12-23 meses, aproximando-se do valor em 2011, 94\%. Todavia, nota-se o aumento da cobertura, onde foram $72 \%$ em < 1 ano e $75 \%$ entre $12-23$ meses em 2001 e, em $2015,92 \%$ e $90 \%$ respectivamente (Tabela 3).

Em Teresina constatou-se que a cobertura vacinal foi de $75,1 \%$. De acordo com os pais, os motivos principais para o atraso vacinal foram: falta de vacina, descuido, adoecimento, indisponibilidade de tempo e distância da UBS. Além disso, crianças que não frequentam a puericultura tiveram $71 \%$ mais propensão de atraso vacinal quando comparadas as que frequentam, o que elucida a maior e melhor alternativa: garantia e fortalecimento das ações de APS (FERNANDES AC, et al., 2015).

Dentre a avaliação do crescimento, o peso deve ser observado com atenção pelo profissional de saúde por tratar-se de uma medida que sofre variações e indica aspectos biológicos e sociais (HANZEN IP, et al., 2019). Quanto à quantidade pesadas < 1 ano e entre 12-23 meses, 2001-2015, constatou-se uma queda. $\mathrm{Em}<1$ ano, em 2003, o total de crianças era 26.874, sendo dentre estas $96 \%$ pesadas. Em contrapartida, 2015, os < 1 ano totalizavam 14.102 sendo pesados apenas $82 \%$. Corroborando com isso, infantes $12-23$ meses, em 2003, 95\% foram pesadas, todavia, 2015, apenas 78\% (Tabela 4). 
Além do exposto acima, observa-se que no estudo realizado com 4.078 crianças, de municípios do Rio Grande do Norte e Santa Catarina, apenas $52 \%$ foram pesadas na UBS de abrangência (PICCINI RX, et al., 2007).

$\mathrm{Na}$ avaliação do peso, um dos fatores identificados é a presença de desnutrição infantil (LOPES AF, et al., 2019). Como um reflexo da melhoria na escolaridade materna, poder aquisitivo, acesso aos cuidados de saúde e condições de saneamento, a prevalência de crianças brasileiras, $<5$ anos, com desnutrição crônica apresentou diminuição de aproximadamente $50 \%$ entre 1996-2007. Já o Norte, com prevalência de 14,7\% de desnutrição, o dobro da média nacional, evidencia a hipótese de que a manutenção de fatores determinantes pode contribuir para que tal região não tenha acompanhado o declínio nacional, podendo-se destacar: dificuldades de deslocamento, indicadores socioeconômicos desfavoráveis e região concentrar a maior parcela da população indígena nacional (LOPES AF, et al., 2019; ARAÚJO TS, et al., 2016).

Contrapondo-se a esse cenário temos que, em Rio Branco, houve um declínio na quantidade de crianças acometidas com desnutrição dentre as pesadas. Em 2001 a prevalência de desnutrição, dentre as pesadas $<1$ ano e $12-23$ meses, foi de $7 \%$ e $26 \%$ respectivamente. Já em 2015 , aproximou-se de $1 \%$ em ambas as idades (Tabela 4).

No Jordão-Acre, estudo com infantes de 0-59 meses, retratou a prevalência da desnutrição como sendo de 7,3\%. Nesse cenário, é importante destacarmos que esta cidade foi classificada, em 2006, como o município com maior estimativa de risco de desnutrição no Brasil (ARAÚJO TS, et al., 2016).

Em contrapartida, no Maranhão, estudo com 956 crianças, 6-59 meses, demonstrou prevalência de infantes com diagnóstico de magreza acentuada e magreza de 0,3\% e 1,5\% respectivamente, considerada uma prevalência baixa (LOPES AF, et al., 2019).

Ao analisarmos a percepção materna quanto ao estado nutricional do filho, relata-se que este é fator determinante para a escolha do padrão dos cuidados que irão definir tanto a alimentação quanto hábitos alimentares, piorando o problema por falta de intervenção precoce (FREITAS TP, et al., 2015).

Apesar do declínio nos casos de desnutrição, em Rio Branco, até os dias de hoje ocorrem internações por má nutrição. Nos < 1 ano, evidenciou-se que em todos os anos ocorreram internações cujo motivo principal era desnutrição. Analisando ano a ano, o resultado encontrado é satisfatório por apresentar um declínio de internações, tendo em 2001 sido 8 e em 2019 metade (Tabela 5).

Porém, mesmo com queda nas internações, nota-se uma taxa de mortalidade considerada elevada. Dentre os 19 anos estudados, ocorreram óbitos por desnutrição em 8 deles. Tendo, portanto, variado a taxa de mortalidade entre 16,67-37,5, em que o último ano que apresentou taxa expressiva foi 2016 (Tabela 5).

Dentre as consequências da desnutrição temos a anemia que está relacionada à maior morbimortalidade infantil e neonatal, diminuição da capacidade física de trabalho e produtividade. Mesmo diante das estratégias governamentais de controle e prevenção da anemia vemos que a deficiência de ferro é a principal causa de anemia em $<2$ anos, como consequência das inadequadas práticas alimentares, crescimento acelerado e infecções recorrentes, especialmente em crianças em vulnerabilidade (OLIVEIRA CS, et al., 2016).

Estudo realizado anteriormente em Rio Branco, com 150 crianças de 11-14 meses, relacionou, como maior risco para a anemia, os seguintes aspectos: não ser filho único, escolaridade materna, desnutrição, introdução tardia de alimentos ricos ou promotores da absorção de ferro e deficiência de vitamina A e B12. A prevalência de desnutrição foi equivalente a $7,7 \%$, o que superou a estimada pela PNDS (Pesquisa Nacional de Demografia e Saúde) em crianças $<11$ meses $(4,8 \%)$ e esteve fortemente associada à anemia. A frequência de anemia foi de $22,7 \%$, deficiência de ferro de $75,8 \%$ e anemia por deficiência de ferro englobou $19,5 \%$ (OLIVEIRA CS, et al., 2016).

No levantamento de causas de internações em Rio Branco, destacou-se o decréscimo significativo na quantidade de internações por anemia e na taxa de mortalidade desta. Em 2001, 2002, 2006 e 2007 foram os anos em que ocorreram maiores internações por anemia, tendo sido 9, 10, 10 e 17 respectivamente. Já a taxa de mortalidade esteve nula de 2012-2019 (Tabela 5). 
Tabela 4 - População, pesadas, desnutridas e média das varáveis, de < 1 ano e 12-23 meses, de Rio Branco, 2001-2019.

\begin{tabular}{|c|c|c|c|c|c|c|c|c|c|c|}
\hline \multirow[b]{2}{*}{ Ano } & \multicolumn{5}{|c|}{$<1$ ano } & \multicolumn{5}{|c|}{ Idade $12-23$ meses } \\
\hline & Crianças & Pesada & $\begin{array}{c}\text { Pesadas } \\
(\%)\end{array}$ & Desnutrida & $\begin{array}{l}\text { Desnutrida } \\
(\%)\end{array}$ & Crianças & Pesada & $\begin{array}{c}\text { Pesadas } \\
\text { (\%) }\end{array}$ & Desnutrida & $\begin{array}{c}\text { Desnutrida } \\
(\%)\end{array}$ \\
\hline 2001 & 37.966 & 35.077 & $92 \%$ & 2486 & $7 \%$ & 43.777 & 40.022 & $91 \%$ & 10449 & $26 \%$ \\
\hline 2002 & 28.020 & 26.564 & $95 \%$ & 1829 & $7 \%$ & 32.768 & 30.830 & $94 \%$ & 7361 & $24 \%$ \\
\hline 2003 & 26.874 & 25.708 & $96 \%$ & 2014 & $8 \%$ & 28.713 & 27.252 & $95 \%$ & 5756 & $21 \%$ \\
\hline 2004 & 26.349 & 25.075 & $95 \%$ & 1809 & $7 \%$ & 28.903 & 27.206 & $94 \%$ & 4873 & $18 \%$ \\
\hline 2005 & 29.501 & 25.495 & $86 \%$ & 1064 & $4 \%$ & 31.591 & 26.651 & $84 \%$ & 2793 & $10 \%$ \\
\hline 2006 & 30.457 & 27.195 & $89 \%$ & 668 & $2 \%$ & 31.479 & 27.244 & $87 \%$ & 1957 & $7 \%$ \\
\hline 2007 & 27.820 & 25.464 & $92 \%$ & 552 & $2 \%$ & 28.561 & 25.456 & $89 \%$ & 1286 & $5 \%$ \\
\hline 2008 & 27.539 & 24.727 & $90 \%$ & 434 & $2 \%$ & 29.890 & 26.247 & $88 \%$ & 985 & $4 \%$ \\
\hline 2009 & 26.140 & 22.160 & $85 \%$ & 316 & $1 \%$ & 26.310 & 21.704 & $82 \%$ & 625 & $3 \%$ \\
\hline 2010 & 20.143 & 16.042 & $80 \%$ & 140 & $1 \%$ & 21.750 & 17.223 & $79 \%$ & 471 & $3 \%$ \\
\hline 2011 & 19.004 & 14.766 & $78 \%$ & 159 & $1 \%$ & 18.769 & 14.083 & $75 \%$ & 201 & $1 \%$ \\
\hline 2012 & 19.344 & 16.094 & $83 \%$ & 197 & $1 \%$ & 19.499 & 15.926 & $82 \%$ & 243 & $2 \%$ \\
\hline 2013 & 22.215 & 18.236 & $82 \%$ & 196 & $1 \%$ & 23.237 & 18.423 & $79 \%$ & 238 & $1 \%$ \\
\hline 2014 & 20.365 & 16.670 & $82 \%$ & 186 & $1 \%$ & 21.070 & 16.392 & $78 \%$ & 280 & $2 \%$ \\
\hline 2015 & 14.102 & 11.585 & $82 \%$ & 103 & $1 \%$ & 15.256 & 11.917 & $78 \%$ & 94 & $1 \%$ \\
\hline Média & 24.856 & 22057 & $87 \%$ & 810 & $3 \%$ & 26.771 & 23105 & $85 \%$ & 2507 & $9 \%$ \\
\hline
\end{tabular}

Fonte: FREITAS TF, et al., 2020; dados extraídos do DATASUS. 
Tabela 5 - Internações e taxa de mortalidade anual de $<1$ ano com desnutrição, anemia, diarreia, pneumonia e internações por diarreia e IRA em < 2 anos, de Rio Branco, 2001-2019.

\begin{tabular}{|c|c|c|c|c|c|c|c|c|c|c|}
\hline \multirow{2}{*}{ Ano } & \multicolumn{2}{|c|}{ Desnutrição < 1 ano } & \multicolumn{2}{|c|}{ Anemia < 1 ano } & \multicolumn{2}{|c|}{ Diarreia $<1$ anos } & \multirow{2}{*}{$\begin{array}{c}\text { Diarreia < } \\
\text { anos }\end{array}$} & \multicolumn{2}{|c|}{ Pneumonia $<1$ ano } & \multirow{2}{*}{$\begin{array}{c}\begin{array}{c}\text { IRA }<2 \\
\text { anos }\end{array} \\
\text { Internação }\end{array}$} \\
\hline & Internação & $\begin{array}{c}\text { Taxa } \\
\text { mortalidade }\end{array}$ & Internação & $\begin{array}{c}\text { Taxa de } \\
\text { mortalidade }\end{array}$ & Internação & $\begin{array}{c}\text { Taxa de } \\
\text { mortalidade }\end{array}$ & & Internação & $\begin{array}{c}\text { Taxa } \\
\text { mortalidade }\end{array}$ & \\
\hline 2001 & 8 & 37,5 & 9 & 0 & 20 & 0 & 11.898 & 287 & 2,44 & 14063 \\
\hline 2002 & 8 & 0 & 10 & 0 & 7 & 0 & 8.362 & 269 & 1,12 & 9680 \\
\hline 2003 & 3 & 33,33 & 7 & 14,29 & 5 & 0 & 8.201 & 419 & 0,95 & 10215 \\
\hline 2004 & 6 & 0 & 5 & 0 & 0 & 0 & 8.975 & 438 & 1,6 & 9805 \\
\hline 2005 & 5 & 0 & 5 & 0 & 1 & 0 & 9.697 & 635 & 0,94 & 11378 \\
\hline 2006 & 2 & 0 & 10 & 0 & 0 & 0 & 8.782 & 792 & 1,39 & 10417 \\
\hline 2007 & 7 & 0 & 17 & 33,33 & 0 & 0 & 5.948 & 626 & 1,83 & 8900 \\
\hline 2008 & 7 & 28,57 & 6 & 0 & 0 & 0 & 5.534 & 247 & 5,26 & 8279 \\
\hline 2009 & 5 & 0 & 4 & 0 & 0 & 0 & 4.561 & 274 & 2,92 & 8011 \\
\hline 2010 & 2 & 0 & 4 & 25 & 15 & 6,67 & 3.588 & 106 & 5,66 & 5529 \\
\hline 2011 & 6 & 33,33 & 5 & 20 & 19 & 0 & 2.979 & 132 & 2,27 & 4770 \\
\hline 2012 & 5 & 20 & 6 & 0 & 36 & 0 & 3.453 & 145 & 4,14 & 4796 \\
\hline 2013 & 4 & 25 & 3 & 0 & 9 & 0 & 3.126 & 81 & 2,47 & 5369 \\
\hline 2014 & 2 & 0 & 2 & 0 & 9 & 0 & 2.945 & 79 & 2,53 & 4609 \\
\hline 2015 & 6 & 16,67 & 2 & 0 & 9 & 11,11 & 1.890 & 54 & 1,85 & 3130 \\
\hline 2016 & 4 & 25 & 1 & 0 & 5 & 0 & 0 & 53 & 9,43 & 0 \\
\hline 2017 & 1 & 0 & 5 & 0 & 5 & 0 & 0 & 56 & 0 & 0 \\
\hline 2018 & 2 & 0 & 5 & 0 & 4 & 0 & 0 & 47 & 2,13 & 0 \\
\hline 2019 & 4 & 0 & 2 & 0 & 2 & 0 & 0 & 70 & 7,17 & 0 \\
\hline
\end{tabular}

Fonte: FREITAS TF, et al., 2020; dados extraídos do DATASUS. 
A importância do AM como prática ideal de nutrição infantil para o pleno crescimento e desenvolvimento da criança, promoção e redução da morbimortalidade infantil, melhoria da qualidade de vida, é demasiadamente conhecida (OLIVEIRA CS, et al., 2016; CIAMPO LA, et al., 2016). Nos primeiros seis meses o leite materno é o alimento capaz de atender todas as particularidades do metabolismo e o contato físico entre mãe-filho proporciona transferência de carinho e afeto, bilateralmente (SANTOS CS, et al., 2007).

A prevalência do AM ainda não coincide com a preconizada pela OMS, que recomenda como forma de alimentação exclusiva até 6 meses, e associada a outros alimentos até 2 anos ou mais. Constata-se isto devido o início do desmame precoce, mesmo com $71 \%$ das crianças brasileiras iniciando a amamentação no primeiro dia de vida (CARDOSO LO, et al., 2008).

O desmame precoce é um importante problema de saúde em todo o mundo e ocorre por diversos fatores, como idade materna, primiparidade, baixa escolaridade, trabalho, urbanização, tabagismo, falta de incentivo e deficiências na atenção à saúde (CIAMPO LA, et al., 2016). Além disso, o peso ao nascer também é apontado como fator, dado que a criança apresenta dificuldades no estabelecimento do AM por imaturidade na frequência e pressão de suç̧ão, além de longos períodos de internação (CARVALHO MJ, et al., 2018).

Uma das formas de se promover o AM é a puericultura. Por meio dela é possível enfatizar a importância desta prática para criança e mãe, evitar introdução precoce de outros líquidos e alimentos, orientar e esclarecer dúvidas, consolidar o conhecimento sobre o tema e eliminar fatores que possam dificultar a consolidação (FONSECA MM, et al., 2015). É possível que os tabus da família estejam direcionando o padrão alimentar das crianças, sobressaindo-se às orientações recebidas pelas mães na consulta de puericultura (FREITAS TP, et al., 2015). A prática de oferta de água, chás, sucos e leites de outras origens é algo corriqueiro na população e fortemente enraizado na cultura, sendo encorajada por figuras mais experientes (CARVALHO MJ, et al., 2018).

Importante salientar que a visita puerperal atua como protetor na manutenção da prática do AME. O apoio profissional por meio da escuta, atenção humanizada, esclarecimento das dúvidas, orientação da técnica correta de amamentação e do suporte no enfrentamento das possíveis dificuldades, contribui para que a mulher inicie e continue o AME (CARVALHO MJ, et al., 2018). Quanto ao AME constatou-se crescimento e predominância nas crianças < 4 meses, 2001-2015 em Rio Branco. Em 2001, a população < 4 meses era 12.625, onde destes, foram avaliados $98 \%$, cujos que se encontravam em AME corresponderam apenas à $64 \%$. Por outro lado, em 2015 , evidenciou-se AME presente em $74 \%$ da população total desta idade. Diante disto, podemos afirmar que a cobertura de AME cresceu dentre os 15 anos estudados, porém ainda se mantém baixa (Tabela 6).

Tabela 6 - População, avaliados e em AME de < 4 meses, em Rio Branco, 2001-2015.

\begin{tabular}{cccccc}
\hline \multirow{2}{*}{ Ano } & \multicolumn{5}{c}{ Infantes < + meses } \\
\cline { 2 - 5 } & População total & Avaliadas & Avaliadas (\%) & AME & AME (\%) \\
\hline 2001 & 12625 & 12402 & $98 \%$ & 7944 & $64 \%$ \\
2002 & 9306 & 9206 & $99 \%$ & 5916 & $64 \%$ \\
2003 & 8718 & 8646 & $99 \%$ & 5842 & $68 \%$ \\
2004 & 8447 & 8378 & $99 \%$ & 5847 & $70 \%$ \\
2005 & 10324 & 10228 & $99 \%$ & 7276 & $71 \%$ \\
2006 & 10307 & 10177 & $99 \%$ & 7415 & $73 \%$ \\
2007 & 8927 & 8847 & $99 \%$ & 6502 & $73 \%$ \\
2008 & 9134 & 9056 & $99 \%$ & 6824 & $75 \%$ \\
2009 & 8287 & 8229 & $99 \%$ & 6028 & $73 \%$ \\
2010 & 6498 & 6460 & $99 \%$ & 4845 & $75 \%$ \\
2011 & 6120 & 6065 & $99 \%$ & 4683 & $77 \%$ \\
2012 & 5794 & 5704 & $98 \%$ & 4337 & $76 \%$ \\
2013 & 6787 & 6606 & $97 \%$ & 5097 & $77 \%$ \\
2014 & 6056 & 5882 & $97 \%$ & 4576 & $78 \%$ \\
2015 & 4496 & 4365 & $97 \%$ & 3251 & $74 \%$ \\
\hline
\end{tabular}

Fonte: FREITAS TF, et al., 2020; dados extraídos do DATASUS. 
Todavia, ao observarmos as práticas de AM na população de Jordão, nota-se que a média de AME foi de apenas 45 dias. Tendo assim, as crianças que tiveram AME pelo período mínimo de 30 dias ou mais, apresentado prevalência de déficit de altura/idade $40 \%$ menor do que aquelas desmamadas anteriormente (ARAÚJO TS, et al., 2016).

Outro estudo com evidências quanto ao AME foi realizado em Rio Branco e mostrou que a mediana de idade do AME e do desmame foram de 120 e 150 dias respectivamente. Corroborando, portanto, com a elevada taxa de crianças < 4 meses em AME na cidade (OLIVEIRA CS, et al., 2016). Por saber que crianças que vivem em condições ambientais inadequadas são mais propensas às doenças infecciosas, levantou-se dados quanto às internações e taxa de mortalidade por doenças prevalentes em infantes 0-2 anos que são consideradas evitáveis quando há eficácia da puericultura (OLIVEIRA CS, et al., 2016).

Dentre a quantidade de infantes 0-2 anos internados por diarreia, 2001-2015, houve uma queda expressiva, pois em 2001 as internações por diarreia foram 11.898 e em 2015 apenas 1.890. Observa-se que quanto os $<1$ ano, houve diminuição entre 2001-2019, sendo a quantidade de internações 20 e 2 respectivamente. A taxa de mortalidade em $<1$ ano nos últimos quatro anos manteve-se nula (Tabela 5). Outro levantamento realizado em Rio Branco, retratou que 27\% dos avaliados, 11-14 meses, apresentaram quadro de diarreia nos 15 dias que antecederam a pesquisa (OLIVEIRA CS, et al., 2016). Corroborando temos estudo realizado com 164 infantes de Londrina, evidenciando que 20,9\% da população apresentou problemas nutricionais e gastrointestinais (ABE R e FERRARI RA, 2008).

Outra afecção comum em < 2 anos que sofrem com não adesão ao AME, desnutrição, alimentação inadequada e falha na puericultura, vem a ser a IRA que compreende patologias como pneumonia, bronquite, bronquiolite aguda e resfriado comum (FERLINI R, et al., 2016). Sendo que, tais agravos respiratórios, constituem a principal causa de internações e a segunda de mortalidade infantil (ABE R e FERRARI RA, 2008). Em avaliação anterior dos infantes de Rio Branco, constatou-se que $70 \%$ das crianças apresentaram quadro de tosse dentre os 15 dias antecedentes a entrevista (OLIVEIRA CS, et al., 2016). Porém, em Londrina, apenas $14,3 \%$ da população estudada apresentou problemas respiratórios (ABE R e FERRARI RA, 2008).

No estudo em vigor, dentre < 2 anos, 2001-2015, o pico de IRA foi em 2001 totalizando 14.063 internações. Em contrapartida, em 2015 ocorreu menos internações, 3.130. Houve declínio expressivo nas internações por IRA, no entanto, ainda permanece alto quando avaliamos que está classificada dentre as principais causas de óbitos evitáveis em crianças. Além do mais, 2001-2019, houve diversas variações quanto a taxa de mortalidade da pneumonia. Em 2001, tal valor esteve em 2,44, já em 2019 sofreu aumento para 7,17. No entanto, a taxa de mortalidade esteve maior em 2016, 9,43 (Tabela 5).

Observa-se que há necessidade de ampliar e garantir o acesso à assistência de qualidade por meio da puericultura que, nas ações de prevenção, incentiva o AME e amplia a cobertura vacinal, condições essenciais para que o RN adquira anticorpos, diminuindo o risco de morbidade causada pelas doenças prevalentes, especialmente as afecções respiratórias (ABE R e FERRARI RA, 2008).

\section{CONCLUSÃO}

A implantação da puericultura trouxe resultados positivos ao avaliar a saúde infantil no Brasil. Porém muitas UBS não usam tal ferramenta adequadamente, o que prejudica a melhora dos indicadores de saúde infantil, tornando assim um país heterogéneo. Concernente a isto, evidenciou-se que no estado do Acre também há uma diversidade entre seus munícipios e que, Rio Branco, como capital, ainda deixa a desejar no quesito de cobertura da puericultura. Tal falha carrega consigo grandes consequências, como poucas crianças com calendário vacinal atualizado, baixa cobertura vacinal, precário acompanhamento do peso, alta taxa de mortalidade por desnutrição, baixa prevalência de AME, muitas internações por IRA e alta mortalidade por pneumonia. Vê-se necessário que os órgãos competentes necessitam avaliar a cobertura de puericultura das UBS de Rio Branco e implementar políticas públicas que incentivem tal prática por parte da equipe de saúde e pela população que, mesmo em 2020, carrega muitos mitos, tabus e crenças indevidas consigo, para ocorrer melhora dos indicadores de saúde infantil. 


\section{REFERÊNCIAS}

1. ABE R, FERRARI RA. Puericultura: problemas materno-infantis detectados pelos enfermeiros numa unidade de saúde da família. Revista Mineira de Enfermagem, 2008.

2. ARAÚJO TS, et al. Desnutrição infantil em um dos municípios de maior risco nutricional do Brasil: estudo de base populacional na Amazônia Ocidental Brasileira. Revista Brasileira de Epidemiologia, 2016; 19: 554-566.

3. BRASIL. Agenda de compromissos para a Saúde Integral da Criança e Redução da Mortalidade Infantil. Ministério da Saúde, Brasília, 2005.

4. BRASIL. Departamento de Informática do Sistema Único de Saúde do Brasil (DATASUS). Ministério da saúde.

5. CAMPOS RM, et al. Consulta de enfermagem em puericultura: a vivência do enfermeiro na Estratégia de Saúde da Família. Revista da Escola de Enfermagem da USP, 2011; 45: 566-574.

6. CARDOSO LO, et al. The impact of implementation of the Breastfeeding Friendly Primary Care Initiative on the prevalence rates of breastfeeding and causes of consultations at a basic healthcare center. Jornal de Pediatria, 2008; 84: 147-153.

7. CARVALHO MJ, et al. Primeira visita domiciliar puerperal: uma estratégia protetora do aleitamento materno exclusivo. Revista Paulista de Pediatria, 2018; 36: 66-73.

8. CIAMPO LA, et al. O Programa de Saúde da Família e a Puericultura. Ciência \& Saúde Coletiva, 2006; 11: $739-743$.

9. CIAMPO LA, et al. Tendência secular do aleitamento materno em uma unidade de atenção primária à saúde maternoinfantil em Ribeirão Preto, São Paulo. Revista Brasileira Saúde Materno Infantil, 2016; 6: 391-396.

10. CUNHA CL, et al. O uso de serviços de atenção primária à saúde pela população infantil em um estudo do nordeste brasileiro. Caderno Saúde Coletiva, Rio de Janeiro, 2013; 21: 115-120.

11. FALBO BC, et al. Estímulo ao desenvolvimento infantil: produção do conhecimento em enfermagem. Revista Brasileira de Enfermagem, 2012; 65: 148-154.

12. FERLINI R, et al. Characteristics and progression of children with acute viral bronchiolitis subjected to mechanical ventilation. Revista Brasileira de Terapia Intensiva, 2016; 28: 55-61.

13. FERNANDES AC, et al. Análise da situação vacinal de crianças pré-escolares em Teresina (PI). Revista Brasileira de Epidemiologia, 2015; 18: 870-882.

14. FONSECA MM, et al. Socio-demographic profile and breastfeeding promotion of nursing professionals of the Family Health Strategy. Revista de Enfermagem Referência, 2015; 5: 85-92.

15. FREITAS TP, et al. Fatores associados à subestimação materna do peso da criança: um estudo de base populacional. Revista de Nutrição, 2015; 28: 397-407.

16. HANZEN IP, et al. Diagnósticos, intervenções e resultados de enfermagem para subsidiar a consulta de enfermagem à criança. Enfermagem foco, 2019.

17. LOPES AF, et al. Perfil nutricional de crianças no estado do Maranhão. Revista Brasileira de Epidemiologia, 2019; 22.

18. OLIVEIRA CS, et al. Anemia e deficiência de micronutrientes em lactentes atendidos em unidades básicas de saúde em Rio Branco, Acre, Brasil. Ciência \& Saúde Coletiva, 2016; 21: 517-530.

19. PICCINI RX, et al. Efetividade da atenção pré-natal e de puericultura em unidades básicas de saúde do Sul e do Nordeste do Brasil. Revista Brasileira de Saúde Materno Infantil, v. 7, p. 75-82, 2007.

20. SANTOS CS, et al. Fatores que interferem na transição alimentar de crianças entre cinco e oito meses: investigação em serviço de puericultura do Recife, Brasil. Revista Brasileira de Saúde Materno Infantil, 2007; 7: 373-380.

21. SANTOS ME, et al. Avaliação dos marcos do desenvolvimento infantil segundo a estratégia da atenção integrada às doenças prevalentes na infância. Escola Anna Nery, 2010; 14: 591-598.

22. SOCIEDADE BRASILEIRA DE PEDIATRIA. Tratado de Pediatria. Editora Manole, Barueri, 2017.

23. TAVARES MN, et al. Consulta de enfermagem em puericultura na estratégia saúde da família: revisão integrativa. Nursing, São Paulo, 2019; 22: 3144-3149.

24. TEJADA CA, et al. Crises econômicas, mortalidade de crianças e o papel protetor do gasto público em saúde. Ciência \& Saúde Coletiva, 2019; 24: 4395-4404.

25. VASCONCELOS VM, et al. Puericultura em enfermagem e educação em saúde: percepção de mães na estratégia saúde da família. Escola Anna Nery, 2012; 16: 326-331.

26. VIEIRA GO, et al. Fatores associados ao uso da Caderneta de Saúde da Criança em uma cidade de grande porte do nordeste brasileiro, 2009. Ciência \& Saúde Coletiva, 2017; 22: 1943-1954.

27. VITOLO MR, et al. Frequência de utilização do serviço público de puericultura e fatores associados. Jornal de Pediatria, 2010; 86: 80-84. 